\title{
E-Assessment Cloud Solution: Architecture, Organization and Cost Model
}

\author{
http://dx.doi.org/10.3991/ijet.v8iS2.2783 \\ Marjan Gusev, Sasko Ristov, Goce Armenski, Goran Velkoski, and Krste Bozinoski \\ Ss. Cyril and Methodius University, Skopje, Macedonia
}

\begin{abstract}
Several challenges address the existing eAssessment systems, such as accurate evaluation, security and data privacy, performance, scalability etc. Our focus in this paper is on e-Assessment scalability and performance. We have developed a new SOA architecture of a cloud hosted e-Assessment system to achieve sustainable performance as a highly scalable and elastic solution. The proposed organization solution is based on three modules (subsystems): management module, reporting module, and assessment module. The built in logic enables an efficient environment where minimum resources will be utilized during exploitation. The proposed system organization ensures that the assessment module, as active subsystem for each assessment, will work with much smaller data compared to the centralized one. The cost model developed for this solution analysis this cloud based solution against the existing e-Assessment web application. Therefore, we expect that this solution will have several benefits, like overall cost reduction and better performance.
\end{abstract}

Index Terms-eAssessment, Cloud Computing, Load Balancing, Performance.

\section{INTRODUCTION}

Leading organizations in e-Learning community were focused on creation of a common technical framework recently. Few detailed frameworks were developed and consensus was made that e-Learning system architecture should be based on service-oriented architecture (SOA) [1]. SOA was widely adopted paradigm, and it was introduced as the architecture for the next generation of eLearning systems [2]. Main benefit of this architecture is the ability to mix together services from different eLearning frameworks.

e-Assessment is a part of integrated e-Learning system dedicated to assess the student knowledge. Armenski and Gusev [3] proposed interoperable e-Assessment Framework introducing SOA. SOAP and REST are two pervasive SOA paradigms for exchanging messages between web services and applications. The latter is more efficient in terms of network bandwidth utilization, round-trip latency, implementation complexity and message processing overhead than the former [4], [5]. Castillo ete al. [6] give detailed comparison of both protocols.

However, both implementations cannot offer sustainable performance since e-Assessment system requires huge amount of unpredictable resources only in appropriate small periods during the e-Assessments, while in most of the time this system requires predictable much less resources. Using server-cluster architecture to create scalable and highly available solutions [7] will only partially solve a performance peak problem since it is hard to be managed and administered. On the other hand, the software solution has to be prepared for scalability.

Cloud computing is a new technology trend which provides computing resources in addition to software in form of a service [8]. Cloud providers offer on-demand infinite elastic resources using virtualization [9]. Cloud computing addresses a set of services providing scalable, QoS guaranteed, normally personalized, inexpensive computing platforms on demand [10]. Cloud billing model is similar to basic utilities billing models, i.e. it is proportional to the amount of usage. Therefore, cloud computing is admissible for educational institutions, especially in present economic crisis, and there has been a lot of advices to universities to turn their interest towards cloud computing services [11], [12], [13]. However, cloud computing degrades the SOA web service performance to 71 to $73 \%$ compared to traditional "bare metal" web service hosting infrastructure with the same hardware resources [14].

Universities can benefit if they migrate their e-Learning systems onto cloud [15] since the cloud offers good resources flexibility and scalability, storage, computational requirements and network access, and most important lower cost [16]. Cloud lowers the disk space requirements, and enables newest software versions and monitoring of the installation progress in each of the cloud service models, i.e. Infrastructure-as-a-Service (IaaS), Platform-as-aService (PaaS) and Software-as-a-Service (SaaS) [17]. Our proposed architecture offers a solution for each cloud service layer from IaaS to SaaS. Caminero et al. [18] present how to harness cloud technologies for e-Learning purposes in order to achieve ease of use, scalability, and reduced power consumption. However, if the universities migrate their e-Learning systems on the cloud, they must ensure that the users can access appropriate resources across systems without breaking the copyright of the resource [19]. In this paper we propose an architecture of eAssessment system that can be hosted onto the cloud and use infinite cloud resources dynamically to reduce the costs and to provide better performance.

The rest of the paper is organized as follows: Section II presents several development phases from traditional paper and pencil testing to modern e-Assessment SOA systems. The next Section III presents the challenges of a modern e-Assessment system. Our proposal for architecture and organization of e-Assessment system to be hosted on the cloud is presented in Section IV. The e-Assessment cloud solution software engineering is elaborated in Section V. Section VI presents the cost model of our eAssessment cloud solution, comparing it with the existing on-premise system. We discuss the benefits of the new proposed cloud e-Assessment architecture and organization in Section VII. Related work in the area of scalable 
cloud architecture and e-Assessment solutions are presented in Section VIII. The final Section IX is devoted for conclusion and future work.

\section{BACKGROUND}

The conventional testing process is organized as paper and pencil testing. Evolution of computer technology enabled realization of variable-form testing approaches that utilize the interactive ability of a computer to administer a set of items that is determined at examination time, rather than a predetermined set of items [20]. Luecht and Sireci [21] present a brief history of computer based testing or eTesting, although the most common used term lately is computer assisted assessment for processes that cover any use of computers in the process of assessing knowledge, skills and abilities of individuals [22].

The infants of e-Learning, i.e. teaching machines date from the 19th century. Although plenty of teaching machines were patented [23], they were hugely criticized during the 1960s despite huge interests about them. Rapid development of microprocessors enabled personal computers to be used in classrooms which is introduced as the first generation of e-Assessment systems. Monolithic assessment applications are the first generation of eAssessment systems [24]. Although the solution was widely adopted, it contained architectural problems. Usually the e-Learning systems were exclusively dedicated to single course or features for communication with external systems were excluded. Additionally, re-usability of existing services was unavailable.

The second generation of e-Assessment system introduced modular system architecture with primary goal for sanitization of architectural issues. Modular architecture allowed homogeneous interconnected components to be used. Additionally, it was suggested that component management, customization and re-usability will be fairly simple. Therefore, pedagogical diversity satisfying various needs can be achieved.

Dagger et al. [2] analyze the evolution of e-Assessment and the whole e-Learning processes via three different generations, as presented in left part of Figure 1. They have analyzed the required methods, standards and technologies for each of the first two generations and have set up recommendations for the next generation, here referred as third generation.

The e-Learning system growth is exponential [16]. Wide acceptance of e-Assessment systems introduced system scalability as emerging challenge that affect the overall QoS. Therefore, Armenski and Gusev [3] proposed that the next, i.e. the third generation of e-Assessment system should be based on SOA architecture. Scalability feature of SOA architecture is major factor for its widely adoption. Additionally, SOA enables inter-operable communication among various systems. Existence of educational service enabled simple reuse in the creation of new eAssessment systems.

The third generation, i.e. SOA oriented e-Assessment systems are still in use. But, issues like resource provisioning, optimal resource utilization and overall system cost still exist. Therefore, the next generation eAssessment systems must incorporate remedies and mitigations strategies for these issues. Section III describes the main challenges of modern e-Assessment systems.

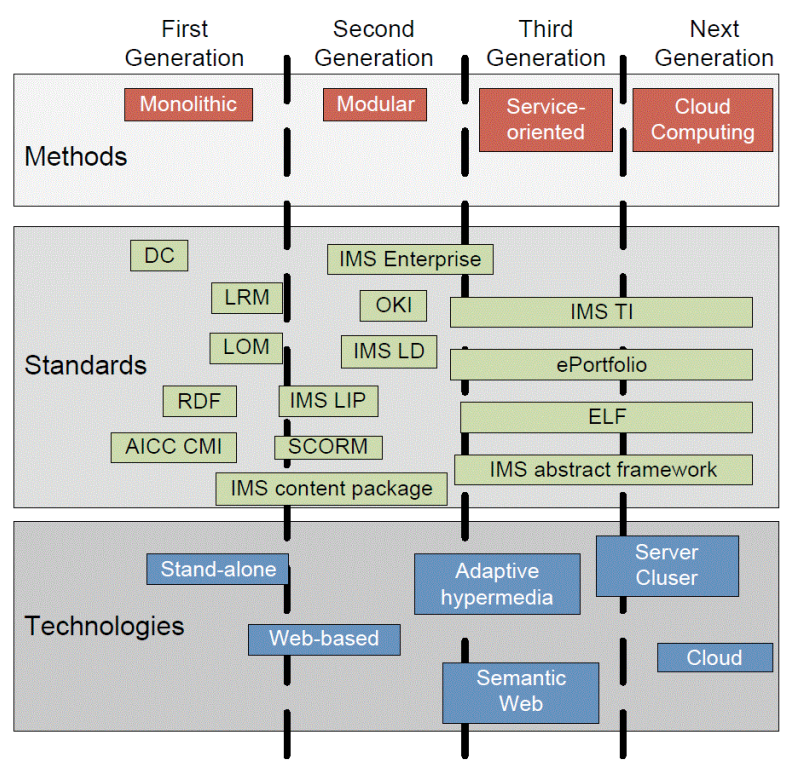

Figure 1. Four generations of e-Assessment and e-Learning systems

\section{TOWARDS TO THE FOURTH GENERATION}

In this section we describe several challenges of the third generation of e-Assessment and the necessity to introduce a new Fourth generation of e-Assessment systems which we call Eco-systems.

\section{A. Abbreviations and Acronyms}

The main e-Assessment challenges are described in this section. Each e-Assessment system must provide accurate and objective assessment as the traditional assessment. Additionally, the system must be reliable and available during the assessments in order to finish the assessment in a proper time.

Assessment results have significant importance for proper student evaluation and introduce increased trustworthiness among the teacher and students via the eAssessment system. However, increased load by increasing the number of assessments and assessed students usually decreases the system performance or even disables it.

e-Assessment reliability is another important challenge. Situations that require additional re-assessment due to any kind of assessment obstructions are unacceptable. These scenarios are almost impossible to be fully avoided, but need to be mitigated deploying redundancy to single point of failure and removing bottlenecks. Even more, particular e-Assessment failure should not affect the others simultaneous assessments.

All participants in e-Assessment process (questions' authors, teachers, managers, system developers and students) suffer from some risks [25]. E-Assessment systems store sensitive data such as student personal data, assessment results and questions for each test. Data privacy and data integrity are imperative requirements for each eassessment system. Additionally, the system must provide authorization and authentication both for students and teachers in order to segregate their access rights. Question banks are another sensitive part of e-Assessment systems and have to be managed according to security policy. Finally, assessment results' confidentiality must be provided. 


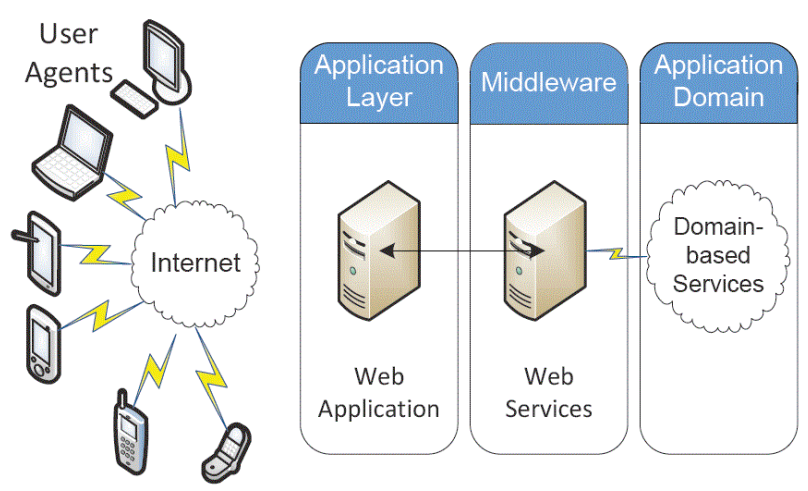

Figure 2. The third generation e-Assessment system architecture [26]

e-Assessment systems must provide fast response after each student answer since the assessment time is limited. e-Assessment performance depends on the total number of enrolled students. The most important factor that impacts the performance is the varying number of assessed students and concurrent assessments. All these requirements introduce a necessity to implement elastic and scalable system for e-Assessment.

The third generation e-Assessment system architecture consists of three subsystems, i.e. Application Layer, Middleware and Application Domains as depicted in Figure 2.

There are three main user roles in e-Assessment system: teachers, students and administrators. The users in teacher role manage the e-Assessment system functions: creation and administration of questions and answers in the system, staring test generation, testing, analysis of results, etc. The students belong to a role that can take the exams and view their results. The administrator role is dedicated to those who administer the system managing with users, courses, exams, tests, configuration etc.

The main problem in this architecture is lack of scalability. For example, to satisfy the growing demand by increased number of students, the administrator of the system will need to upgrade the server infrastructure and enable its robustness.

There are two possible scenarios for resource allocation. The first scenario is to dedicate enough resources to host the e-Assessment system capable to handle the assessment of all enrolled students in one moment. This solution will be very expensive and the overall system will be underutilized most of the time. Also the total number of enrolled students varies during time generating additional scaling problems. The second scenario is to predict the average number of students that are concurrently assessed and to dedicate enough resources to host the e-Assessment system as it can handle the assessment of predicted number of students in one moment. Although this scenario is less expensive, the solution will still be over-utilized or underutilized most of the time.

\section{B. Eco-systems}

In this paper we define a specific organization and solution that will represent the fourth generation of eAssessment systems called eco-systems. Eco-systems are treated as biological living organisms. Dong et al. [27] described the resource provisioning in the cloud as birth and death of the organism. The infants of the fourth generation systems use server clusters [7] in order to gain better performance, optimal resource utilization and re- duce the overall costs. As the cloud computing paradigm emerged, the next generation of e-Assessment systems will be hosted on the cloud. Figure 1 presents the methods, standards and technologies for the fourth generation of e-Assessment and e-Learning.

This generation has added value to the previous generations for the technologies that implement scalability, virtualization, elasticity and interoperability between different systems (including legacy systems) required to become a cloud solution. Used methods refer to introducing cloud computing solution, where all required functionalities are offered as a service. Although development of standards is still hot research topic, several of them have been acknowledged to be accepted for the new generation, like IMS Learner Information Package (IMS LIP) and public and private information (PAPI) for learners (PAPI learner) for user modeling and personalization, IMS Question and Test Interoperability (IMS QTI) for exchange of content and IEEE Learning object metadata (LOM) or Dublin Core Metadata for exchange of metadata.

SOA provides an environment for computing the services, while the cloud provides an environment that offers the services of computing. The combination of SOA and cloud computing also provides potentially transformative opportunities [28].

Here we propose an architecture and organization that can be hosted on the cloud capable to provide fully realtime scalable solution. It is also SOA based architecture which uses all benefits of the cloud.

\section{Cloud E-Assessment Architecture}

In this section we propose an e-Assessment system cloud architecture and organization. The solution is designed to provide sustainable performance with minimal costs for cloud hosting resources.

\section{A. System Overview}

The cloud approach based on demand is not a new idea. However, as they say, the devil is in implementation, so here we address series of implementation details, not just presenting the model specification and service description within the proposed architecture.

Armenski and Gusev [24] present a new high level of abstraction of the architecture of so-called ultimate eAssessment system, as presented in Figure 3. The main accent is set on intra-domain and inter-domain interoperability, although both approaches to build a standalone system or a cloud solution can be build based on this approach. The innovation that this system defines is introduction of a broker module with purpose to communicate to different Learning Management Systems (LMSs) and acts a role of system service orchestrator using the service registry, a form of Universal Description Discovery and Integration (UDDI) approach with descriptions about available service.

In addition to the conventional three layered architecture the offered system architecture model [24] defines common services layer, e-Assessment services layer and composite services layer (Broker). FREMA project [29] defines most of the services in the e-Assessment layer and in common services layer.

The new proposed e-Assessment system organization model consists of three subsystems, i.e. the Management, Assessment and Reporting subsystems, determined by 


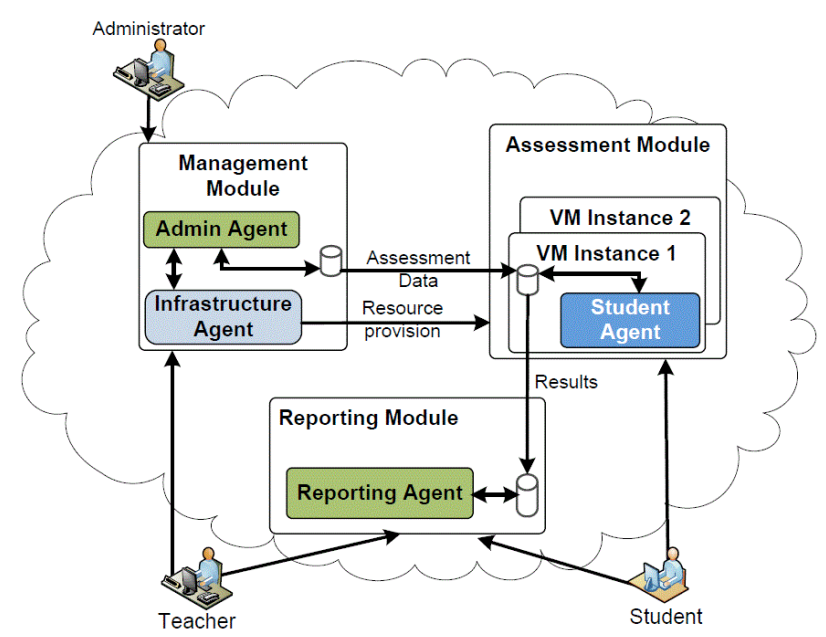

Figure 3. e-Assessment system organization

working mode performance and resource requirements, as presented in Figure 4.

e-Assessment system works in two different modes, the Active Mode, when there is at least one active assessment and the Inactive Mode when there isn't any active assessment. Inactive Mode resource requirements are predictable since it depends mostly on the total number of all students and teachers. It can be easily determined if the load is simulated with some performance tester and the parameters of the previous years are used. Active mode resource requirements are unpredictable since they depend not only from the total number of students and teachers, but mostly from the concurrent number of assessments and assessed students.

In this paper we explain the implementation details of the Broker module allocated in Figure 3. We introduce four agents that are part of a Broker: Admin Agent, Infrastructure Agent, Student Agent and Reporting Agent, as depicted in Figure 4. Next sections give detailed explanation of their purpose and features.

\section{B. Management Module}

Management module is the core of the e-Assessment system. It manages all resource provisioning in the cloud. This module can be hosted on a bare metal server or on a virtual machine (VM) with constant resources since it manages the system when it is in Inactive Mode. It is always active to provide users, courses, questions, exams, authentication, authorization etc.

The management role is realized with two agents: Admin Agent and Infrastructure Agent as depicted in Figure 5.

1) Admin Agent: The Admin Agent is the most versatile part of the system which provides the e-Assessment content administration, data replication to the Student Agent in Assessment Module, authentication and authorization services, and assessment scheduling. The Admin Agent is service oriented and contains several parts:

- LMS - LMS is part of e-assessment system used for courses and knowledge management. It communicates with the Scheduler for assessment timetable

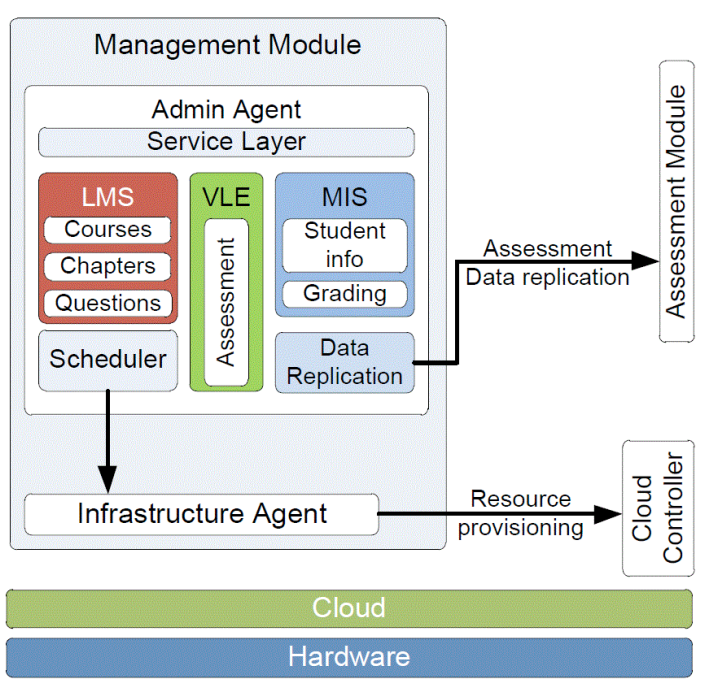

Figure 4. Management module with Admin Agent and Infrastructure Agent

creation and the Virtual Learning Environment (VLE) subsystem. The scheduler communicates with the Infrastructure agent for resource provisioning information delegation.

- VLE - VLE is used for distant learning and assessment. Through this subsystem of e-assessment system, students can easily access to materials for each assessment. However this is also the subsystem responsible for assessment question banks definition and allocation for each assessment. Based on information from this subsystem the scheduler can supply the infrastructure agent with the much needed assessment information such as data bank size for each scheduled assessment, number of students and so on. Additionally, the VLE communicates with the Data Replication subsystem in order to define the parts of data needed to be replicated for Assessment module nodes.

- Management Information System (MIS) - Student information and grading management is MIS responsibility. This subsystem is used for gathering student assessment results and the much needed reporting about them.

2) Infrastructure Agent: The Infrastructure Agent communicates with Scheduler from Admin Agent to create and shut down the instances in the Assessment Module providing the optimal resource utilization according to the number of assessed students and reducing the overall cost for renting the hardware resources.

\section{Reporting Module}

The main idea in our new e-Assessment system is dividing the results of the exams in the new separate module, i.e. Reporting Module. After each assessment, the data is replicated into this module. Then Reporting Agent provides the exam results to both the teachers and students. Data privacy and security are obliged for this part of the system because of the present sensitive data. 


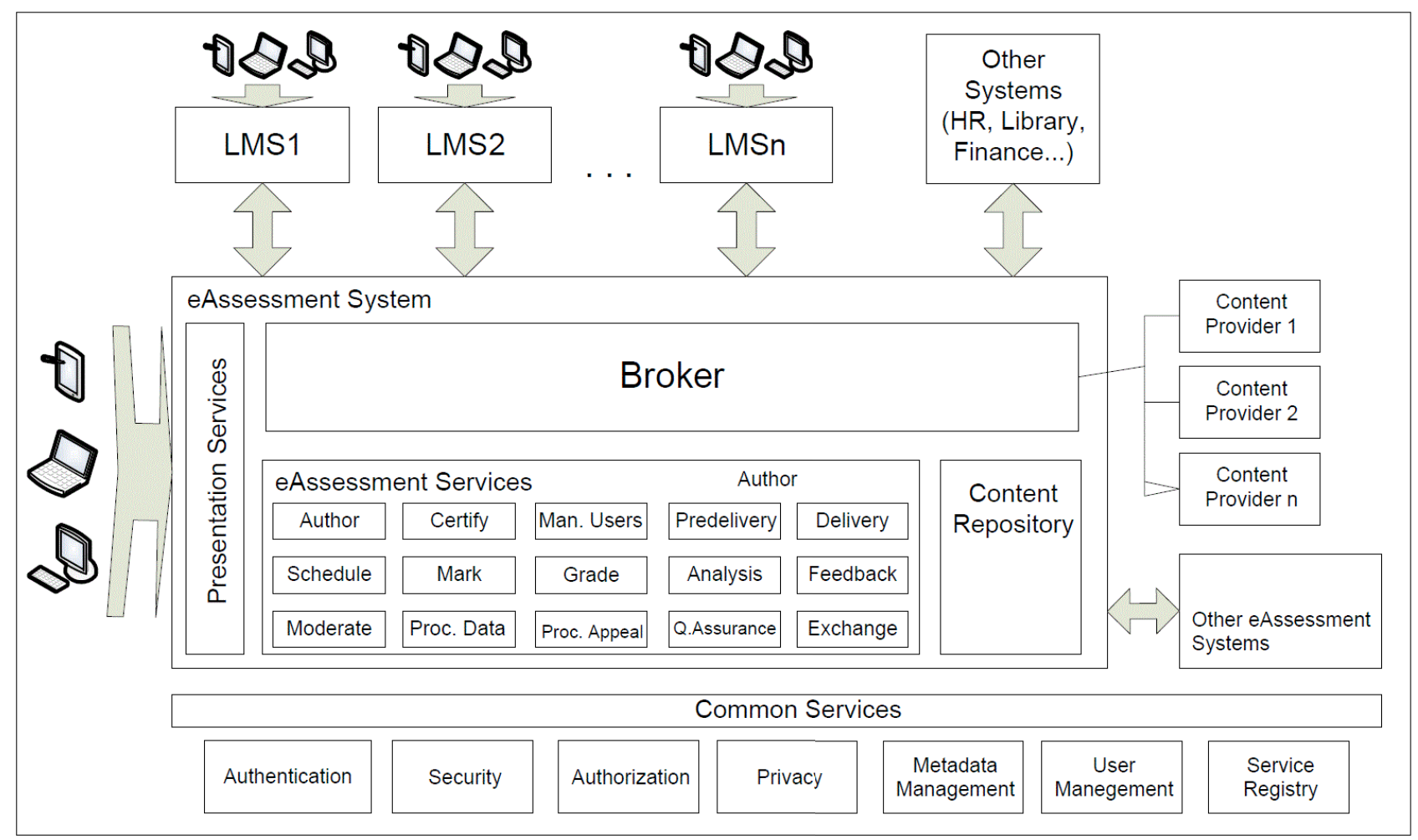

Figure 5. Architecture of an ultimate e-Assessment System [24]

\section{Assessment Module}

Assessment module is active only when e-Assessment system is in active mode. It is devoted for assessments and its load varies depending on the number of assessments and assessed students. Therefore, we propose this subsystem to be hosted on the cloud and to be dynamically allocated with resources.

We introduce resource allocation policy that determines required resources based on the number of students for particular assessment. Moreover, it hosts only small amount of data required for particular e-Assessment, i.e. teacher(s) and enrolled students of the course, exam questions and answers and other similar required data. This makes the system more efficient providing better overall performance.

The data in this module is determined by the course and the number of students taking the exam. Therefore the Student Agent is placed in this module to receive necessary assessment data from Admin Agent and send the results of the assessment to the Reporting Agent to store them in the main reporting database and to be published for the students and the teachers. Figure 6 depicts the Assessment module architecture and Student Agent with in.

Similar to the Admin Agent, the Student Agent is service based. This subsystem is constructed from the same integrated parts as Management Agent, i.e. LMS, VLE and MIS.

Although the same sub systems are used, Student Agent operates on significantly smaller data and has only assessment access rights. Working on relatively smaller database clearly improves the overall e-Assessment performance since each query works on smaller data set.

Student Agent based LMS module is used for course student information gathering only. One agent is invoked for each course and this is then used for e-assessment via the VLE subsystem and question bank LMS. MIS subsystem is used as manager to keep the assessment results temporarily. The main function is to keep temporary the results until they are saved within the main database reporting replication.

\section{Design of a Cloud E-Assessment Model}

In order to facilitate the development of this fourth generation e-Assessment system based on cloud computing, in this section we present the model of cloud eAssessment system with organization of VM instances that host the previously described modules and the sequence diagram that presents activities related to provision and usage of cloud resources.

\section{A. Organization of VM instances}

The model of cloud e-Assessment initially requires one VM instance called Administration Node with goal to hosting the Management module and another VM instance called Reporting Node with goal to hosting the Reporting module, as presented in Figure 7. Administration Node dynamically instantiates and shuts down additional VMs, identified as Assessment Node.

The Administration Node hosts the management module. The specified functions in this module can be accessed by administrators and teachers. The module services communicate with cloud controller for resource provisioning via the infrastructure agent and to assessment module via admin agent. The Reporting Node contains tools to access the database for preview and analysis of e- Assessment results and its functions can be accessed by all authorized users.

The dynamic allocation of cloud resources is initially specified by the number of students applied for assessments. 


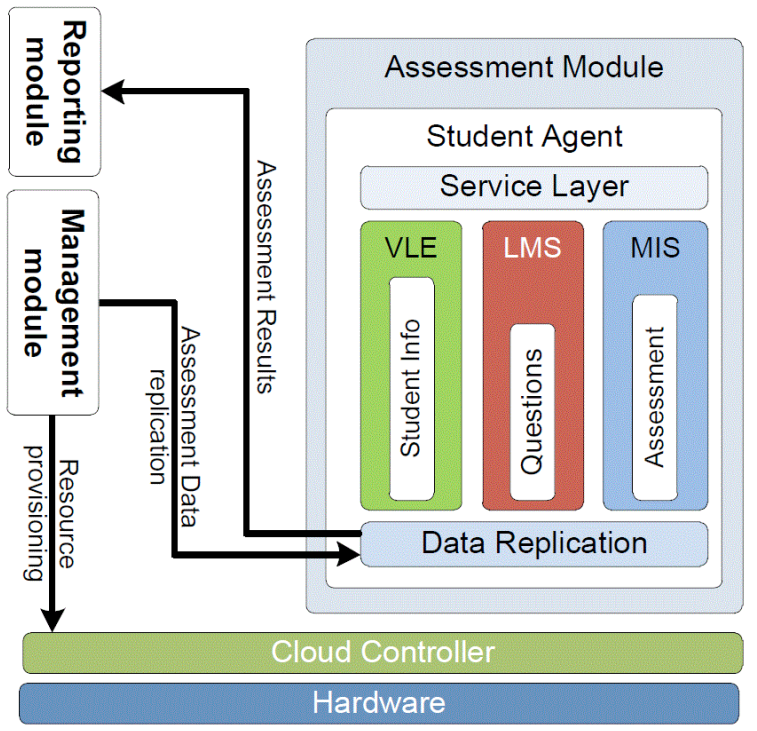

Figure 6. Assessment module with Student Agent

The Assessment Node hosts the assessment module and is accessed by authorized students and teachers. These instances are dynamically created and closed as required by the Management module via the infrastructure agent and are usually managed by the system administrator.

\section{B. Sequence Diagram for Cloud Resources Provision}

Figure 8 presents the sequence diagram for the eAssessment model from aspect how it communicates with cloud, i.e. how the activities for resource provision and releasing are asked by the modules and how the cloud controller instantiates and shuts down corresponding virtual machines with assessment module.

Teacher's interaction with the e-Assessment system consists of two activities: the first is content and grade authoring, and the second is test scheduling. We are not interested in the former since it represents common application scaffolding problem. However, we focus on the latter since it is a baseline to optimize resource utilization. That is, the e-Assessment performance directly depends on the number of concurrent students enrolled in the scheduled assessment.

Students' interaction with the e-Assessment system consists also of two activities: preview of results and testing process. The former mostly consists of database scan and search operations. These resource requirements are predictive and therefore we model all these functions to be hosted on a separate VM for reporting node. The latter has major impact on the system performance due to complex and massive query executions. Data write operations are realized during the assessment and they are stored in the corresponding VM instance identified as Assessment Node.

The process of test scheduling consists of several steps. The teacher defines the course by specifying the questions in corresponding knowledge database and number of students that can apply for assessment. Subsequently Admin Agent adds a new event in the Scheduler component which concludes the process of test scheduling where user interaction is necessary. Event data consists of the previously submitted user data. The Scheduler component trig-

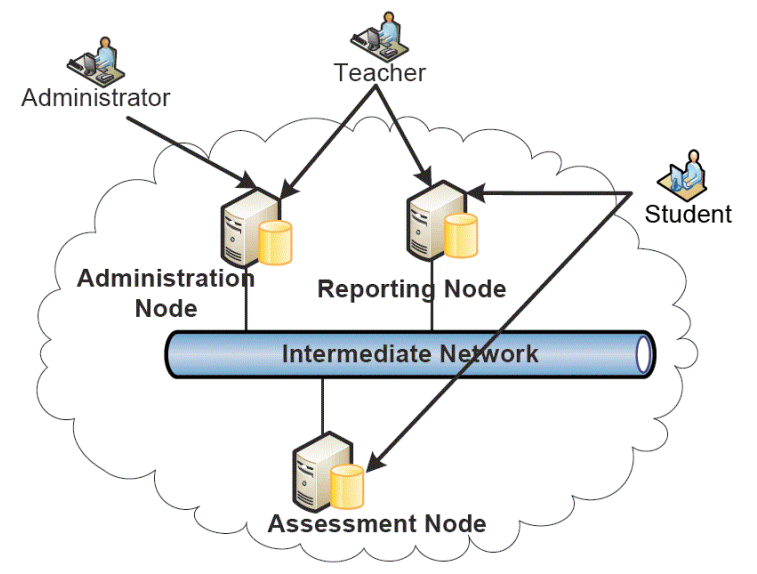

Figure 7. VM instances organization for cloud e-Assessment system

gers the previously stored event at the specified time interval before the assessment. The number of students'value stored in the event is forwarded to the Infrastructure Agent in order to determine the required resources for the Student Agent VM instance. Further on, the Infrastructure Agent sends appropriate request to the Cloud Controller to provide the necessary resources and instantiate new VM instance. When Assessment Module is activated, the Data Replication component of Admin Agent sends necessary data chunks to the Data Replication Component of the new Assessment Module. Data replication component of Assessment Module initialize the secondary database with assessment data. Hereby the test scheduling action is completed.

After the assessment scheduling, the students should authenticate to the system. Access token is endorsed for each active student. Additionally, each student's token is published in the active Assessment Module. Token publishing is constrained only to Student Agents dedicated for courses where the student is enrolled. Successful token creation provides new access token and address of modules where token is published.

Further on, the students take the assessment and after the assessment time has elapsed, Data Replication transfers the results to Reporting Module. At the end, the Infrastructure Agent releases the VM instance that hosted the corresponding Assessment module.

\section{COST MODEL}

This section presents the cost model of our eAssessment cloud solution and compares it with the existing e-Assessment on-premise solution used at our University.

There are several methods and modeling tools to quantitatively compare the cost of leasing cloud resources to that of purchasing and using a dedicated server system. For example, Walker [30] has analyzed mainly the real cost of a CPU hour and its impact on overall resources. Li et al. [31] address the Total Cost of Ownership (TCO) and utilization cost considering the elastic cloud features and virtualization approach. Greenberg [32] analyzes and calculates the cost of a cloud, as a research problem in data center networks. Durke [33] argues why cloud computing will never be free. 
SPECIAL FOCUS PAPER

E-Assessment Cloud Solution: ARChitecture, ORganization AND Cost Model

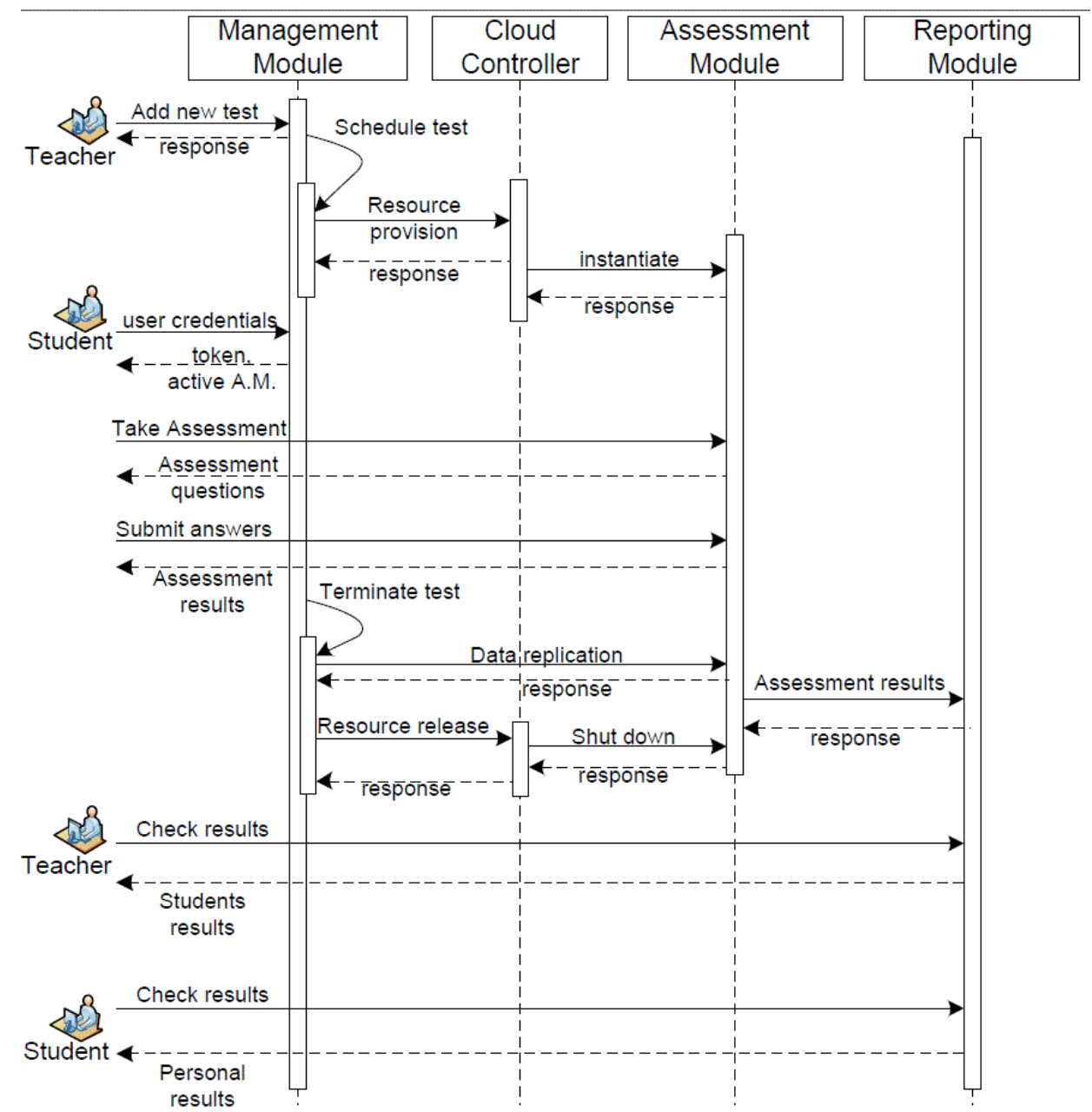

Figure 8. Sequence diagram for e-Assessment model

To build the cost model of our e-Assessment cloud solution we will use the method for cost analysis proposed by $\mathrm{Li}$ et al. [31]. The case study is based on the use of 8 labs with capacity of 20 students each at our Department. The experiment addresses a total of 160 potential concurrent users.

\section{A. Estimation of Required Resources}

The existing e-Assessment solution is configured to handle at least 160 concurrent executions of exams for students. However, the problem appears when the eAssessment system generates the tests.

We analyze a situation where the system generates a different test for each student, which requires enormous data processing. For example, the test strategy selects 50 learning objectives and picks one question in all of these areas. If we assume that at least 4 potential candidates for answers are offered per question, than the total data calculations will address at least $160 * 50 * 4=32.000$ database insertions. All data access and computing appear in concurrent threads simultaneously and the server performance degrades severely during the assessment. We make an estimation that a server is capable to handle at least $1 / 4$ of calculated database insertions and activities of the application and web server simultaneously, dimensioning the server to be capable to handle a maximum 16.000 simultaneous concurrent transactions.

In the following analysis we will make further calculations for three cases based on expected minimal (existing situation), nominal (expected after one year) and maximum (expected after 2 years). It also means that the system should be dimensioned double for 320 students having simultaneous exams after one year (nominal case) or 480 students after two years (maximum case).

\section{B. Existing E-Assessment On-premise Solution}

If the solution is self-hosted server based, than the following calculations can estimate the real costs.

Capital expenditure costs (CAPEX) are estimated for the following: 2 servers are used in the existing solution, each capable to handle at least 8.000 simultaneous concurrent accesses, or one server with 2 virtual machines installed on a more capable server that handles 16.000 simultaneous concurrent accesses. Estimated value of two servers or one more advanced server is $15.000 \$$. On top of this we add $2.000 \$$ for other expenses, for example $500 \$$ for network switch, $1.000 \$$ for UPS and $500 \$$ for air conditioning cooling system. In reality we have to add also storage area network, but this demand will be not used in the model since it will also appear for the cloud solution.

Next we calculate the estimated costs for operating expenses OPEX in a 3-year period. The electrical power is estimated to $3 \mathrm{KW}$ for air conditioning cooling system and $2 \mathrm{KW}$ for ICT, i.e. $5 \mathrm{KW}$. The costs for electrical energy will be 3 years $* 365$ days $* 24$ hours $* 5 \mathrm{KW} * 0.12 \$$ per hour $=15.768 \$$. 
The total calculation of CAPEX + OPEX is more than $30.000 \$$ for a 3 -year period for the estimated case that 160 students can have simultaneously the exams in lab. Although we are aware of, in this calculation we do not add maintenance costs, or costs required for technical assistance or administration.

\section{E-Assessment Cloud Solution}

A simplified calculation gives the following estimations. Each exam session takes part only one week per semester, so in a yearly basis, the system is utilized only 2 semesters $* 3$ exams $* 1$ week $=6$ weeks out of 52 , meaning that the nominal utilization value is $11.54 \%$ of the available time. If the exams are scheduled in two weeks to enable more conditions, than the maximum utilization is $23.07 \%$.

Actual real calculation is presented in the following. Currently 30 courses actively use the e-Assessment per semester and we assume an average of 80 students per course to take part in at least three exams (including midterm and final exam). On a year basis it means that the system will handle 2 semesters $* 30$ courses $* 3$ exams $=$ 180 exams, or in a 3 -year period total of 540 exams. The organization of exams usually takes 4 hours per course due to availability of the lab (80 students scheduled in 4 lab hours). Finally, we can calculate a total of 2160 hours requested from the server on a 3-year basis, meaning that the system is utilized only $8.22 \%$ of available time. The predictions are that the number of courses using this system will double after one year (nominal case with $16.43 \%$ ) and triple after two years (maximum case with $24,66 \%$ ), having the maximum case with similar value as in simplified calculation.

In case of a cloud solution there are no capital investment costs CAPEX we calculate only the operating costs OPEX. The lowest available price (Amazon [36]) for virtual machines with 8 cores is $0.52 \$$ per hour and the maximum (Google [35]) is $1.2 \$$ per hour. Therefore costs are estimated to be $1.123 \$$ as minimum or $2.592 \$$ as maximum.

On top of previous costs we have to add costs for Elastic Load Balancer, Elastic Load Balancer Data Processing, Elastic Block Store volume, S3 Storage for WAR File and Access, Bandwidth In and Out, which is estimated to be 3.300\$ for a 3-year period.

Another issue is the cost of hosting the Management and Reporting Module, since it can be realized as dedicated server and appropriate storage area network. Of course, we can choose whether to install it on premise or host it as dedicated server on the cloud. Estimated price for hosting of dedicated server including the communication traffic costs is (Amazon) approx. 2.500\$ to 5.000\$ for a 3 -year period of medium utilization reserved instances with medium CPU processing power and, medium memory demands and medium communication bandwidth.

The calculation of estimated costs of OPEX is in range between $6.923 \$$ as minimum and $10.892 \$$ as maximum.

\section{Cost Comparison}

The cost comparison of the given model is presented in Figure 9. The price is expressed in USD and the cases represent minimal workload with 160 students taking exams simultaneously, nominal with 320 students and maximal with 480 students.

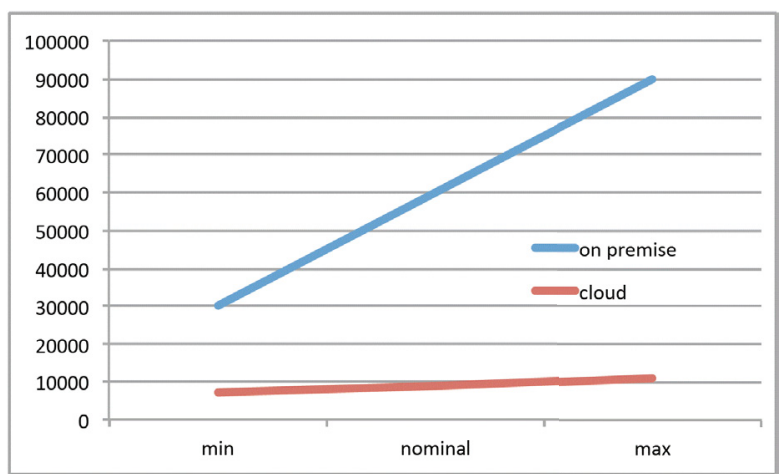

Figure 9. 3-year costs for on-premise and cloud eAssessment solutions

This figure also confirms the advantages of cloud solution over the on-premise. Interestingly, for greater values of utilization of on-premise server we can expect lower costs in the beginning, but higher for increased processing demand. The average value of cost ratio for the given minimal workload is 0.23 if cloud is compared to the onpremise server.

\section{DISCUSSION}

This section discusses all benefits that our new eAssessment solution provides to the Universities. Our solution is scalable and elastic and can be hosted on all three cloud service layers, i.e. IaaS, PaaS and SaaS. Communication with cloud controller is explained in corresponding sequence diagram by the Infrastructure Agent.

The solution is capable to instantiate as many VM instances as enabled by hardware resources in the computer center. It is platform independent and therefore PaaS can be used to implement this solution.

On the other side, this solution can be offered as SaaS services, so the cloud provider will take care about the infrastructure agent and provide relevant number of resources. The e-Assessment solution can be hosted on appropriate IaaS or PaaS cloud service layers. The communication with cloud controllers is scalable and elastic. It instantiates and shuts down VM instances for each eAssessment with particular resources that can provide minimum system performance depending on the number of concurrent students enrolled within the assessment. On the other side, the traditional third generation SOA eAssessment system will be either underutilized or over utilized during the assessment and it is more important that it will be always underutilized when there is not any active assessment which is most of the time during the semester.

We use the fact that particular student will be enrolled in maximum one assessment at a given time and therefore our system will utilize only minimum resources required to provide minimum performance. The traditional "tight" third generation e-Assessment system utilizes constant resources during the assessments, but also before and after them.

Even more, apart from its scalability and elasticity, our solution has another organizational advantage compared to third generation e-Assessment systems providing better performance with the same hardware resources. That is, each Student Agent works with its own database containing only the necessary data for particular e-Assessment. It is much smaller than the third generation e-Assessment 
main database which contains data for all expired assessments in the past.

Our solution is cost effective, i.e. the universities reduce their costs for buying the expensive hardware equipment in advance. Even more, not only that the equipment will be underutilized most of the time, but it will spend a lot of electricity power unnecessary.

\section{RELATED WORK}

The cloud computing paradigm offers many benefits in the whole education, not just for e-Assessment. It stores the educational resources, e-mails, educational applictions and tools, thus reducing the costs and improving the quality of current system of education [15]. Many e-Learning cloud architectures are proposed to achieve this objective.

Masud and Huang [34] present a framework which specifies a number of steps how the universities should adopt the cloud computing. The framework consists of several features: ESaaS (Education Software as a service), Digital Library for easy content download, online storage to maintain students' data, student collaboration, network access to cloud resources, interoperability with different clouds, provisioning and security and privacy.

Our analysis of relevant literature shows that there are neither published cloud e-Assessment solutions nor available developed e-Assessment cloud implementations. Some early efforts are realized in the e-Learning area, or its subset, without specifying the web services or SaaS solutions for e-Assessment. For example, Tu et al. [37] proposed a model for cloud learning platform which can be successfully used to teach the increased number of immigrants with the domestic Chinese language.

\section{CONCLUSION AND Future WORK}

There are several challenges for cloud e-Assessment system that initiate motivation to replace the traditional eAssessment. One of the most important challenge is to survive the peaks when particular number of assessments are taken with particular number of assessed students.

In this paper we propose an architecture of cloud eAssessment system that improves the overall performance and reduces the costs. The contribution can be summarized as three-fold for the proposed architecture:

- Handles loads since it uses as many resources as needed when it is in Active Mode;

- Provides better performance since each Assessment instance works with database chunks instead of huge main database; and

- Reduces the overall cost since it requires only minimum resources for Management Module and Reporting Module when it is in Inactive Mode and also minimizes resources for additional instances in Assessment Module when it is in Active Mode.

This paper refers not only to a cloud architecture, but it also defines the fourth generation of e-Assessment development.

In future we plan a series of experiments to analyze the benefits of the new cloud e-Assessment system compared to the on-premise solution.

\section{ACKNOWLEDGMENT}

This work was partially financed by the Faculty of Computer Science and Engineering at the "Ss. Cyril and Methodius University", Skopje, Macedonia.

\section{REFERENCES}

[1] K. Blinco, J. Mason, N. McLean, and S. Wilson, "Trends and issues in e-learning infrastructure development," Altilab04, Redwood City, California, USA, 2004.

[2] D. Dagger, A. O'Connor, S. Lawless, E. Walsh, and V. Wade, "Serviceoriented e-learning platforms: From monolithic systems to flexible services," Internet Computing, IEEE, vol. 11, no. 3, pp. 28-35, 2007. http://dx.doi.org/10.1109/MIC.2007.70

[3] G. Armenski and M. Gusev, "E-testing based on service oriented architecture," in 10th CAA International Computer Assisted Assessment Conference. Loughborough University, 2006, pp. 17-26.

[4] G. Mulligan and D. Gra canin, "A comparison of soap and rest implementations of a service based interaction independence middleware framework," in Winter Sim. Conf., ser. WSC '09, 2009, pp. 1423-1432.

[5] S.-C. Hu, I.-C. Chen, and Y.-L. Lin, "Designing a restful question bank service in cloud," in Multimedia Technology (ICMT), 2011 International Conference on, july 2011, pp. $5803-5806$.

[6] P. A. Castillo, J. L. Bernier, M. G. Arenas, J. J. M. Guerv'os, and P. Garc'ra-S'anchez, "Soap vs rest: Comparing a master-slave ga implementation," CoRR, vol. abs/1105.4978, 2011.

[7] J.-H. Ho, M.-Y. Luo, and C.-S. Yang, "Building a scalable digital learning system on server clusters," J. Inf. Sci. Eng., vol. 23, no. 3, pp. 803-819, 2007.

[8] M. Armbrust, A. Fox, R. Griffith, A. Joseph, R. Katz, A. Konwinski, G. Lee, D. Patterson, A. Rabkin, I. Stoica et al., "A view of cloud computing," Communications of the ACM, vol. 53, no. 4, pp. 50-58, 2010. http://dx.doi.org/10.1145/1721654.1721672

[9] P. Mell and T. Grance, "The nist definition of cloud computing (draft)," NIST special publication, vol. 800, p. 145, 2011.

[10] L. Wang, J. Tao, M. Kunze, A. Castellanos, D. Kramer, and W. Karl, "Scientific cloud computing: Early definition and experience," in High Performance Computing and Communications, 2008. HPCC'08. 10th IEEE International Conference on. IEEE, 2008, pp. 825-830.

[11] M. Mircea and A. Andreescu, "Using cloud computing in higher education: A strategy to improve agility in the current financial crisis," Communications of the IBIMA, vol. 2011, 2011.

[12] S. Gupta, "Cloud computing in education in current financial crisis," in International Conference on Technology and Business Management March, vol. 26, 2012, p. 28.

[13] D. Chandra and M. Borah, "Cost benefit analysis of cloud computing in education," in Computing, Communication and Applications (ICCCA), 2012 International Conference on. IEEE, 2012, pp. $1-6$.

[14] S. Ristov, G. Velkoski, M. Gusev, and K. Kjiroski, "Compute and memory intensive web service performance in the cloud," in ICT Innovations 2012. Springer Berlin / Berlin Heidelberg, 2013, vol. AISC 257, pp. 215-224.

[15] A. Ghazizadeh, "Cloud computing benefits and architecture in elearning," in Wireless, Mobile and Ubiquitous Technology in Education (WMUTE), 2012 IEEE Seventh International Conference on, 2012, pp. 199-201.

[16] A. Fernndez, D. Peralta, F. Herrera, and J. Bentez, "An overview of e-learning in cloud computing," in W. on Learning Techn. for Educ. in Cloud (LTEC'12), ser. Advances in Intelligent Sys. and Comp., L. Uden et al., Ed. Springer Berlin Heidelberg, 2012, vol. 173, pp. 35-46.

[17] F. Doelitzscher, A. Sulistio, C. Reich, H. Kuijs, and D. Wolf, "Private cloud for collaboration and e-learning services: from iaas to saas," Computing, vol. 91, no. 1, pp. 23-42, Jan. 2011. http://dx.doi.org/10.1007/s00607-010-0106-z

[18] A. Caminero, A. Robles-Gomez, S. Ros, R. Hernandez, R. Pastor, N. Oliva, and M. Castro, "Harnessing clouds for e-learning: New directions followed by uned," in Global Engineering Education Conference (EDUCON), 2011 IEEE, april 2011, pp. $412-416$. http://dx.doi.org/10.1109/EDUCON.2011.5773169 


\section{SPECIAL FOCUS PAPER \\ E-Assessment Cloud Solution: ArChitecture, Organization ANd Cost Model}

[19] Z. He and J. Yue, "Integrating e-learning system based on cloud computing," in Granular Computing (GrC), 2012 IEEE International Conference on, 2012, pp. 1-4.

[20] N. Thompson and D. Wiess, "Computerised and adaptive testing in educational assessment," The transition to computer-based assessment. New approaches to skills assessment and implications for large-scale testing, pp. 127-133, 2009.

[21] R. Luecht and S. Sireci. (2012) A review of models for computerbased testing. [Online]. Available: http://research.collegeboard.org/publications/content/2012/05/revi ew-models-computer-based-testing

[22] N. Sclater and K. Howie, "User requirements of the ultimate online assessment engine," Computers \& Education, vol. 40, no. 3, pp. 285-306, 2003. http://dx.doi.org/10.1016/S03601315(02)00132-X

[23] L. Benjamin, "A history of teaching machines." American Psychologist; American Psychologist, vol. 43, no. 9, p. 703, 1988. http://dx.doi.org/10.1037/0003-066X.43.9.703

[24] G. Armenski and M. Gusev, "The architecture of an ultimate eassessment system," Association for Information and Communication Technologies ICT-ACT, 2009.

[25] N. Barik and S. Karforma, "Risks and remedies in e-learning system," CoRR, vol. abs/1205.2711, 2012.

[26] M. Al-Smadi and C. Gutl, "Soa-based architecture for a generic and flexible e-assessment system," in Education Engineering (EDUCON), 2010 IEEE, april 2010, pp. $493-500$.

[27] B. Dong, Q. Zheng, J. Yang, H. Li, and M. Qiao, “An e-learning ecosystem based on cloud computing infrastructure," in Advanced Learning Technologies, 2009. ICALT 2009. Ninth IEEE International Conference on. IEEE, 2009, pp. 125-127.

[28] Y.Wei and M. Blake, "Service-oriented computing and cloud computing: Challenges and opportunities," Internet Computing, IEEE, vol. 14, no. 6, pp. 72-75, 2010. http://dx.doi.org/10.1109/MIC.2010.147

[29] G. Wills, C. Bailey, H. Davis, L. Gilbert, Y. Howard, S. Jeyes, D. Millard, J. Price, N. Sclater, R. Sherratt et al., "An e-learning framework for assessment (frema)," Assessment \& Evaluation in Higher Education, vol. 34, no. 3, pp. 273-292, 2009. http://dx.doi.org/10.1080/02602930802068839
[30] E. Walker, "The real cost of a CPU hour," Computer, vol. 42, no. 4, pp. 35-41, Apr. 2009. http://dx.doi.org/10.1109/MC.2009.135

[31] X. Li, Y. Li, T. Liu, J. Qiu, and F. Wang, "The method and tool of cost analysis for cloud computing," in Cloud Computing, 2009. CLOUD '09. IEEE International Conference on, 2009, pp. 93100.

[32] A. Greenberg, J. Hamilton, D. A. Maltz, and P. Patel, "The cost of a cloud: research problems in data center networks," SIGCOMM Comput. Commun. Rev., vol. 39, no. 1, pp. 68-73, Dec. 2008. http://dx.doi.org/10.1145/1496091.1496103

[33] D. Durkee, "Why cloud computing will never be free," Queue, vol. 8, no. 4, p. 20, 2010.

[34] M. Masud and X. Huang, "A novel approach for adopting cloudbased e-learning system," in Computer and Information Science (ICIS), 2012 IEEE/ACIS 11th International Conference on, 2012, pp. $37-42$.

[35] Google. "Compute Engine," http://cloud.google.com/pricing/, May, 2013

[36] Amazon. "EC2," http://aws.amazon.com/ec2/, May, 2013.

[37] C.-C. Tu and A.-P. Chen, "Building a learning games network in cloud learning platform based on immigrant education," in $A d$ vances in Social Networks Analysis and Mining (ASONAM), 2011 International Conference on, 2011, pp. 746-750.

\section{AUTHORS}

Marjan Gusev, Sasko Ristov, Goce Armenski, Goran Velkoski, and Krste Bozinoski are with Ss. Cyril and Methodius University, Faculty of Information Sciences and Computer Engineering, Rugjer Boshkovik 16, PO Box 393, 1000 Skopje, Macedonia. Email: \{marjan.gushev, sashko.ristov, goce.armenski, \}@finki.ukim.mk, velkoski.goran@gmail.com, kbozinoski@hotmail.com.

This article is an extended version of a paper presented at the IEEE EDUCON20213 Conference, held from March 13th-15th, 2013, in Berlin, Germany. Submitted 15 May 2013. Published as re-submitted by the authors 25 July 2013. 